\title{
Suppression of conductance in a topological insulator nanostep junction
}

\author{
M. Alos-Palop, ${ }^{1}$ Rakesh P. Tiwari, ${ }^{2}$ and M. Blaauboer ${ }^{1}$ \\ ${ }^{1}$ Delft University of Technology, Kavli Institute of Nanoscience, Department of Quantum Nanoscience, Lorentzweg 1 , \\ 2628 CJ Delft, The Netherlands \\ ${ }^{2}$ Department of Physics, University of Basel, Klingelbergstrasse 82, CH-4056 Basel, Switzerland
}

(Received 19 April 2012; published 29 January 2013)

\begin{abstract}
We investigate quantum transport via surface states in a nanostep junction on the surface of a three-dimensional topological insulator that involves two different side surfaces. We calculate the conductance across the junction within the scattering matrix formalism and find that as the bias voltage is increased, the conductance of the nanostep junction is suppressed by a factor of $\frac{1}{3}$ (independent of the system parameters) as compared to the conductance of a similar planar junction based on a single surface of a topological insulator. We also calculate and analyze the Fano factor of the nanostep junction and predict that the Fano factor saturates at $\frac{1}{5}$, five times smaller than for a Poisson process.
\end{abstract}

DOI: 10.1103/PhysRevB.87.035432

PACS number(s): 73.20.-r, 73.23.-b, 73.40.-c

Experimental demonstration of topological phases in both two-dimensional (such as $\mathrm{HgTe}$ ) and three-dimensional (such as $\mathrm{Bi}_{2} \mathrm{Se}_{3}$ ) compounds with strong spin-orbit interaction ${ }^{1-4}$ has generated a plethora of interest in the physics community. ${ }^{5}$ These compounds are insulating in the bulk (since they have an energy gap between the conduction band and the valence band) but their surfaces support gapless topological excitations. These surface states are topologically protected against nonmagnetic defects by time-reversal symmetry. ${ }^{5}$

In the simplest case, these low-energy excitations of a strong three-dimensional (3D) topological insulator can be described by a single Dirac cone at the center of the two-dimensional Brillouin zone ( $\Gamma$ point). ${ }^{1,3,5}$ Recently, naturally occurring defects such as steplike imperfections $s^{6,7}$ have been studied in the context of topological insulators. Conductance through atomic-scale step defects has been measured, demonstrating that the surface states are protected in spite of such an abrupt defect. $^{6}$ These atomic-sized steps are usually modeled as delta function potential barriers. ${ }^{8}$ In this article, we investigate quantum transport through a nanostep junction involving two different side surfaces of a 3D topological insulator. We predict that the conductance of the nanostep junction is suppressed in the large-energy limit by a factor of $\frac{1}{3}$ (independent of the system parameters) as compared to the conductance of a similar junction based on a single surface of a 3D topological insulator (or a similar junction in graphene). Figure 1(a) shows a schematic of the nanostep junction. The junction is divided into three regions. In regions I $(x<0$ and $z=0)$ and III $(x>0$ and $z=L)$, the surface states lie in the $x-y$ plane, while in region II $(x=0$ and $0 \leqslant z \leqslant L)$, the surface states lie in the $y-z$ plane. A dc bias voltage is applied between regions I and III and a top gate $V_{s}$ controls the carriers in region II. Figure 1(b) shows a schematic of the analogous planar junction, where in all three regions the surface states lie in the $x-y$ plane and a top gate $V_{p}$ is applied to region II. The first junction [Fig. 1(a)] is referred to as a step junction, and the second junction [Fig. 1(b)] as a planar junction in the rest of this article. We use the conceptually transparent scattering matrix formalism ${ }^{9}$ to calculate the transport properties of these junctions. For concreteness, we use typical parameter values of the 3D topological insulator $\mathrm{Bi}_{2} \mathrm{Se}_{3}$ when illustrating our results.
The low-energy effective Hamiltonian for $\mathrm{Bi}_{2} \mathrm{Se}_{3}$ in the basis of four hybridized states of Se and $\mathrm{Bi} p_{z}$ orbitals denoted as $\left\{\left|p 1_{z}^{+}, \uparrow\right\rangle,\left|p 2_{z}^{-}, \uparrow\right\rangle,\left|p 1_{z}^{+}, \downarrow\right\rangle,\left|p 2_{z}^{-}, \downarrow\right\rangle\right\}$ can be written as ${ }^{10}$

$$
H(\mathbf{k})=\epsilon_{0}(\mathbf{k})+\left[\begin{array}{cccc}
\mathcal{M}(\mathbf{k}) & A_{1} k_{z} & 0 & A_{2} k_{-} \\
A_{1} k_{z} & -\mathcal{M}(\mathbf{k}) & A_{2} k_{-} & 0 \\
0 & A_{2} k_{+} & \mathcal{M}(\mathbf{k}) & -A_{1} k_{z} \\
A_{2} k_{+} & 0 & -A_{1} k_{z} & -\mathcal{M}(\mathbf{k})
\end{array}\right],
$$

where $\quad k_{ \pm}=k_{x} \pm i k_{y}, \quad \epsilon_{0}(\mathbf{k})=C+D_{1} k_{z}^{2}+D_{2} k_{+} k_{-}$, $\mathcal{M}(\mathbf{k})=M-B_{1} k_{z}^{2}-B_{2} k_{+} k_{-}$, and $k_{+} k_{-}=k_{x}^{2}+k_{y}^{2}$. Here, $\uparrow(\downarrow)$ stands for up (down) spin and $+(-)$ stands for even (odd) parity. From this three-dimensional Hamiltonian, a straightforward procedure exists to obtain the effective Hamiltonian describing the surface states. ${ }^{11,12}$ The surface states in the $x-y$ plane, for example, are obtained from the three-dimensional wave functions for these surface states (which are exponentially damped in the $z$ direction, with finite skin depth $\lambda$ ) using Eq. (1), followed by imposing the boundary conditions of vanishing wave functions at the two boundaries $(z=0$ and $L)$. For three-dimensional topological insulators $(L \gg \lambda)$, the surface states at the two boundaries are decoupled and the effective Hamiltonian describing the carriers in regions I and III is then given by ${ }^{12,13}$

$$
\mathcal{H}^{x y}=\epsilon_{0}^{x y}+\hbar v_{F}^{x y}\left(\sigma_{x} k_{y}-\sigma_{y} k_{x}\right),
$$

where $\epsilon_{0}^{x y}=C+\left(D_{1} / B_{1}\right) M, \hbar v_{F}^{x y}=A_{2} \sqrt{1-\left(D_{1} / B_{1}\right)^{2}}$ represents the Fermi velocity in the $x-y$ plane, and $\sigma_{x}, \sigma_{y}$, and $\sigma_{z}$ denote the usual Pauli matrices.

Analogously, we obtain the effective Hamiltonian describing the carriers in region II as

$$
\mathcal{H}^{y z}=\epsilon_{0}^{y z}+e V_{s}+\hbar v_{F}^{y z}\left(\sigma_{y} \frac{A_{1}}{A_{2}} k_{z}-\sigma_{z} k_{y}\right),
$$

where $\epsilon_{0}^{y z}=C+\left(D_{2} / B_{2}\right) M$ and $\hbar v_{F}^{y z}=A_{2} \sqrt{1-\left(D_{2} / B_{2}\right)^{2}}$. Solving the Hamiltonian (2), we obtain the eigenstates in regions I and III as

$$
\Psi_{\mathrm{I}(\mathrm{III})}^{ \pm}=\frac{1}{\sqrt{2}}\left[\begin{array}{c}
1 \\
\mp i e^{ \pm i \phi}
\end{array}\right] e^{i k_{y} y} e^{ \pm i k_{x} x},
$$




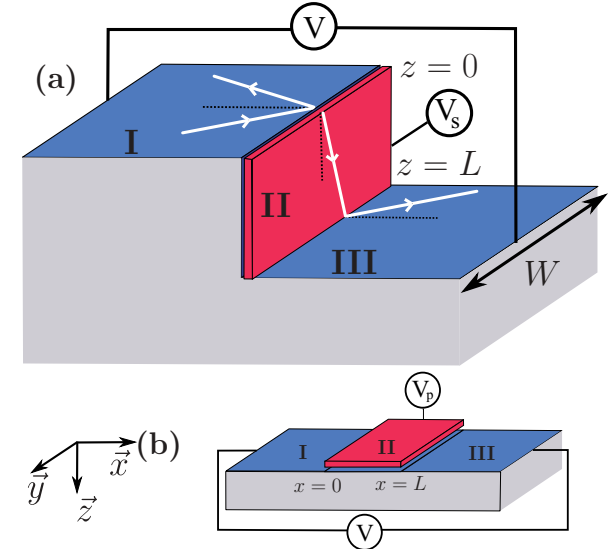

FIG. 1. (Color online) Schematics of the proposed junction. (a) The nanostep junction with two carrier paths indicated (white lines). As the name suggests, the height $L$ of the junction is $\sim 10 \mathrm{~nm}$. (b) Similar junction, involving only one side surface of the topological insulator. See the text for details.

with corresponding energy eigenvalues given by $\epsilon=\epsilon_{0}^{x y}+$ $\hbar v_{F}^{x y} \sqrt{k_{x}^{2}+k_{y}^{2}}$ and $\tan (\phi) \equiv k_{y} / k_{x}$. Similarly, in region II, we obtain

$$
\Psi_{\mathrm{II}}^{ \pm}=\frac{1}{\sqrt{2(1+\sin (\gamma))}}\left[\begin{array}{c}
\mp i \cos (\gamma) \\
1+\sin (\gamma)
\end{array}\right] e^{i k_{y} y} e^{ \pm i k_{z} z},
$$

with corresponding energy eigenvalues $\epsilon=\epsilon_{0}^{y z}+e V_{s}+$ $\hbar v_{F}^{y z} \sqrt{k_{y}^{2}+\left(A_{1}^{2} / A_{2}^{2}\right) k_{z}^{2}}$ and $\tan (\gamma) \equiv A_{2} k_{y} /\left(A_{1} k_{z}\right)$. The $+(-)$ labels of the wave function indicate right (left) traveling carriers in regions I and III, and downwards (upwards) traveling carriers in region II. It should be noted that in general $\epsilon_{0}^{x y} \neq \epsilon_{0}^{y z}$, which implies that the Dirac cone describing the surface states in the $y-z$ plane is shifted by an energy of $\epsilon_{0} \equiv \epsilon_{0}^{y z}-\epsilon_{0}^{x y}$ with respect to the Dirac cone describing surface states in the $x-y$ plane. Furthermore, the Dirac cone describing the excitations in region II has an elliptic cross section $\left(A_{1} \neq A_{2}\right)$. These features are in good agreement with recent electronic-structure calculations of similar systems. ${ }^{14}$

Considering electrons incident from left to right, the total wave function in the different regions can be written as

$$
\begin{array}{ll}
\Psi_{\mathrm{I}}=\Psi_{\mathrm{I}}^{+}+r \Psi_{\mathrm{I}}^{-} & \text {if } \quad x \leqslant 0, \quad z=0, \\
\Psi_{\mathrm{II}}=a \Psi_{\mathrm{II}}^{+}+b \Psi_{\mathrm{II}}^{-} & \text {if } \quad 0 \leqslant z \leqslant L, \quad x=0, \\
\Psi_{\mathrm{III}}=t \Psi_{\mathrm{III}}^{+} & \text {if } \quad x \geqslant 0, \quad z=L .
\end{array}
$$

The reflection and transmission coefficients $r$ and $t$ can be obtained by imposing the boundary conditions under which the current normal to the boundary is conserved. ${ }^{15-17}$ We then find the transmission probability $T \equiv t^{*} t$ of an electron incident on the step junction at a given angle of incidence $\phi$ :

$$
T_{\text {step }}(\phi)=\frac{1}{\cos ^{2}\left(k_{z} L\right)+\sin ^{2}\left(k_{z} L\right) \frac{1}{\cos ^{2}(\phi) \cos ^{2}(\gamma)}},
$$
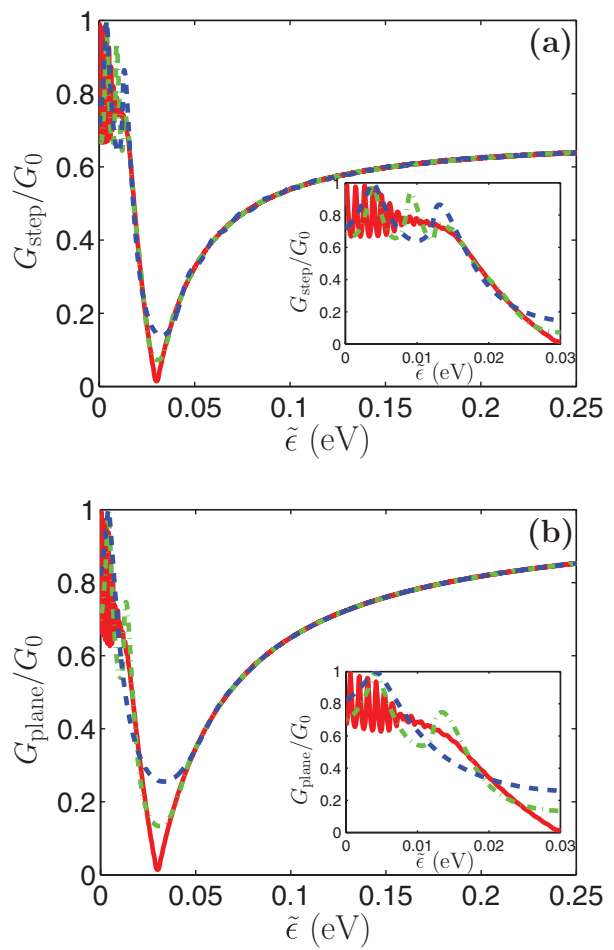

FIG. 2. (Color online) Conductance as a function of energy for (a) the step junction [Eq. (9)] and (b) the planar junction [Eq. (10)]. Insets show the low-energy regime in detail. Different junction widths are plotted. In both plots, the dashed (blue) line denotes $L=50 \mathrm{~nm}$, the dotted-dashed (green) line denotes $L=100 \mathrm{~nm}$, and the solid (red) line corresponds to $L=500 \mathrm{~nm}$. Parameters used are $A_{1}=$ $2.2 \mathrm{eV} \AA, A_{2}=4.1 \mathrm{eV} \AA, \hbar v_{F}^{x y}=4.065 \mathrm{eV} \AA, \hbar v_{F}^{y z}=3.846 \mathrm{eV} \AA$, $\epsilon_{0}^{x y}=0.03 \mathrm{eV}, \epsilon_{0}^{y z}=0.09 \mathrm{eV}, e V_{s}=-0.03 \mathrm{eV}$, and $e V_{p}=0.03 \mathrm{eV}$. See the text for more details.

with

$$
\sin (\gamma)=\kappa \sin (\phi),
$$

$\kappa=\left(v_{F}^{y z} / v_{F}^{x y}\right)\left[\tilde{\epsilon} /\left(\tilde{\epsilon}-\epsilon_{0}-e V_{s}\right)\right] \quad$ and $\quad \tilde{\epsilon} \equiv \epsilon-\epsilon_{0}^{x y}$. Equation (8) is obtained by using conservation of energy and conservation of momentum along the $y$ direction.

The zero-temperature conductance $G_{\text {step }}$ across the step junction is then given by ${ }^{9}$

$$
G_{\text {step }}=G_{0} \int_{0}^{\pi / 2} d \phi T_{\text {step }}(\phi) \cos (\phi) .
$$

Here, $G_{0} \equiv \frac{2 e^{2}}{h} \rho(\tilde{\epsilon}) \hbar v_{F} W, \rho(\tilde{\epsilon})=\tilde{\epsilon} /\left[\pi\left(\hbar v_{F}\right)^{2}\right]$ denotes the density of states, $W$ the sample width, and the integration is over all angles of incidence $\phi$. Figure 2(a) shows the conductance $G_{\text {step }}$ [Eq. (9)] as a function of energy $\tilde{\epsilon}$ for different values of $L$. We observe that the conductance reaches a minimum at $\tilde{\epsilon}=\epsilon_{0}+e V_{s}$, arising from the difference in Dirac point energies in different planes. Beyond this point, the conductance first oscillates and then saturates at large energies. For $\kappa \rightarrow 1$, the conductance $G_{\text {step }}$ reaches a limiting value of $\frac{2}{3}$, independent of barrier width $L$. In the following [see Eq. (11)], we discuss the behavior of the conductance close to this saturation point in more detail.

The transmission probability of the analogous planar junction [see Fig. 1(b)] with a top gate in the middle region is 

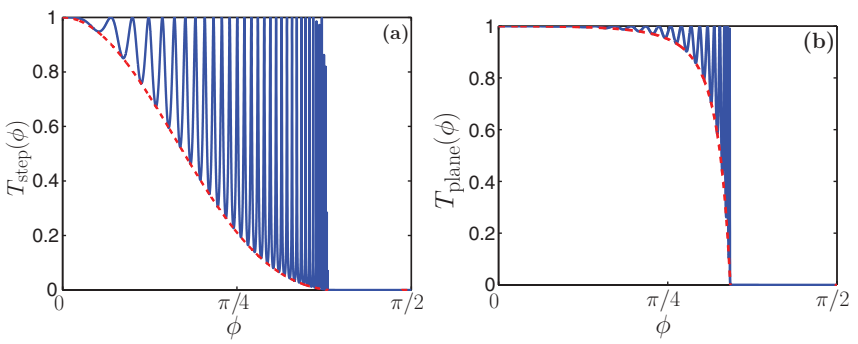

FIG. 3. (Color online) Transmission (a) $T_{\text {step }}(\phi)$ [Eq. (7)] for the step junction and (b) $T_{\text {plane }}(\phi)$ [Eq. (10)] for the planar junction as a function of angle of incidence $\phi$ for $L=100 \mathrm{~nm}$ and $\tilde{\epsilon}=0.25 \mathrm{eV}$. Parameters used are the same as in Fig. 2. The dashed red lines mark the envelopes of the transmission probabilities.

given by ${ }^{18}$

$$
T_{\text {plane }}(\phi)=\frac{1}{\cos ^{2}\left(k_{x}^{\prime} L\right)+\sin ^{2}\left(k_{x}^{\prime} L\right) \frac{\left[1-\sin (\phi) \sin \left(\gamma^{\prime}\right)\right]^{2}}{\cos ^{2}(\phi) \cos ^{2}\left(\gamma^{\prime}\right)}} .
$$

Here, $k_{x}^{\prime}$ represents the $x$ component of the momentum in region II, the energy dispersion is given by $\epsilon=\epsilon_{0}^{x y}+e V_{p}+$ $\hbar v_{F}^{x y} \sqrt{k_{x}^{\prime 2}+k_{y}^{2}}$ and $\gamma^{\prime} \equiv \tan ^{-1}\left(k_{y} / k_{x}^{\prime}\right)$. Figure 2(b) shows the conductance $G_{\text {plane }}$ as a function of energy $\tilde{\epsilon}$ for different values of $L$. As before, the conductance reaches a minimum when $\tilde{\epsilon}=e V_{p}$, and then increases to reach its saturation value $G / G_{0}=1$. For larger energies, the conductance of the step junction is thus suppressed by a factor of $\frac{1}{3}$ compared with planar junctions. We attribute this suppression to the fact that the carriers in the step junction have to change their plane of propagation in region II and part of the current is transmitted into edge states which propagate along the step edge in the $y$ direction. ${ }^{19}$

We now analyze in more detail the difference between the conductance of the step and the planar junction by comparing the denominators in Eqs. (7) and (10). Figure 3 shows the transmission probabilities $T_{\text {step }}(\phi)$ and $T_{\text {plane }}(\phi)$ as a function of the angle of incidence $\phi$. From Fig. 3(a), we see that for the step junction there is a cutoff angle of incidence, which arises from the finite energy and velocity mismatch. This critical angle can be expressed as $\phi_{\text {c,step }}=\sin ^{-1}\left\{\left(v_{F}^{x y} / v_{F}^{y z}\right)\left[\left(\tilde{\epsilon}-\epsilon_{0}-e V_{s}\right) / \tilde{\epsilon}\right]\right\}$. We also see that the conductance of both junctions includes contributions from many resonant modes. Here, a resonant mode is defined as a mode with an angle of incidence for which the transmission $T=1$. The various minima of these resonant modes form an envelope, as shown by the dashed (red) lines in Fig. 3. These envelope functions are obtained from the transmission expressions (7) and (10) by setting $k_{z} L=$ $(2 n+1) \pi / 2$ and $k_{x}^{\prime} L=(2 n+1) \pi / 2$, respectively, with $n$ integer. When the number of resonant modes is large $(n \gg 1)$, a lower bound for the integrated transmission is obtained by integrating over the envelope function. Under these conditions, the conductance $G_{\text {step }}$ of the step junction [Eq. (9)] becomes

$$
\begin{aligned}
G_{\text {step }} / G_{0} & \stackrel{n \gg 1}{\rightarrow} \int_{0}^{\pi / 2} d \phi \cos ^{3}(\phi) \cos ^{2}(\gamma) \\
& =\frac{2}{3}-\frac{2}{15} \kappa^{2} .
\end{aligned}
$$

Similarly, we find for the planar junction

$$
G_{\text {plane }} / G_{0} \stackrel{n \gg 1}{\rightarrow} \frac{\sqrt{\delta}(3 \delta-1)+(\delta-1)^{2} \tanh ^{-1}(\sqrt{\delta})}{2 \delta^{3 / 2}},
$$

where $\delta \equiv \tilde{\epsilon} /\left(\tilde{\epsilon}-e V_{p}\right)$. The suppression of the conductance $G_{\text {step }}$ in the vicinity of the saturation value thus depends on the shift of the Dirac point energies and the ratio of the Fermi velocities in regions I and II. ${ }^{20}$ As final remarks, we note that the effect of the elliptical dispersion in the middle region of the step junction can be incorporated as an effectively wider barrier $\tilde{L}=L\left(A_{2} / A_{1}\right)$ and $\left(A_{2} / A_{1}\right)>1$, compared to the planar junction. The minimum barrier width needed to observe the suppression in the conductance described above is given by the condition for the existence of the first resonant mode in the junction $L=\pi / k_{z}$. In the case of $\mathrm{Bi}_{2} \mathrm{Se}_{3}$, this minimum width is $L \sim 5 \mathrm{~nm}$. For cleaved topological insulators, widths of $L \sim 1 \mathrm{~nm}$ have been reported. ${ }^{21}$

In the remaining part of this article, we investigate the Fano factor of the step junction, which is a measure for the noise suppression in the junction relative to Poisson noise. ${ }^{9}$ Within the scattering matrix formalism, the Fano factor $F$ is defined as

$$
F=\frac{\int_{0}^{\pi / 2} d \phi \cos (\phi) T(1-T)}{\int_{0}^{\pi / 2} d \phi \cos (\phi) T},
$$

where $\phi$ is the angle of incidence of the carriers and $T$ represents the transmission of the junction. Substituting
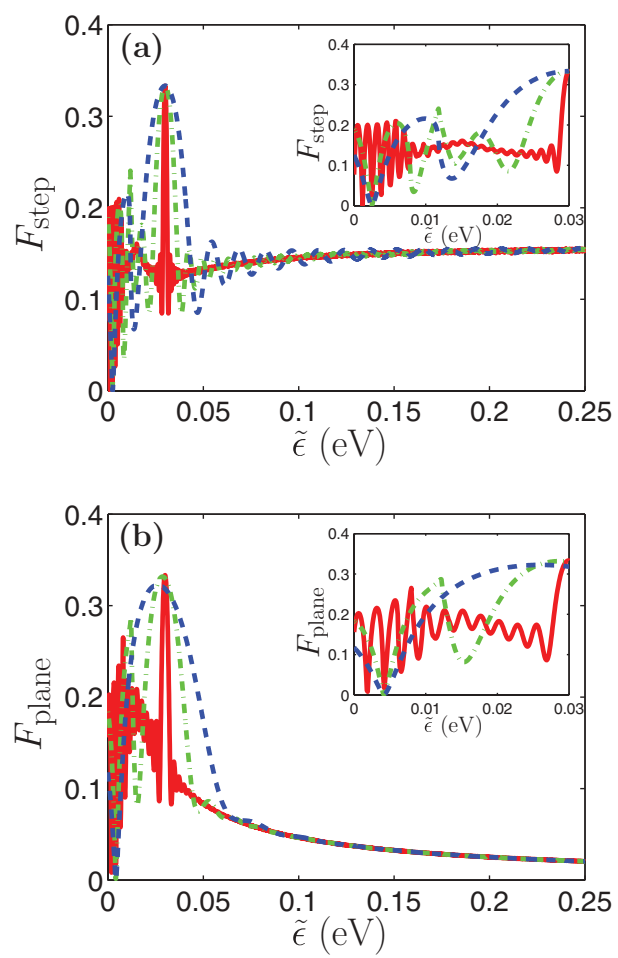

FIG. 4. (Color online) The Fano factor [Eq. (13)] for (a) the step junction and (b) the planar junction as a function of energy $\epsilon$. Insets show the low-energy regime in detail. Different junction widths are plotted. In both plots, the dashed (blue) line denotes $L=50 \mathrm{~nm}$, the dotted-dashed (green) line denotes $L=100 \mathrm{~nm}$, and the solid (red) line corresponds to $L=500 \mathrm{~nm}$. Parameters used are the same as in Fig. 2. 
Eqs. (7) and (10) into Eq. (13) and evaluating the integrals in general leads to lengthy expressions. However, in the limit of a large number of resonant modes $(n \gg 1)$, we find that the Fano factor of the step junction is given $b^{22}$

$$
F_{\text {step }} \stackrel{n \gg 1}{\rightarrow} \frac{1}{5}+\frac{12}{175} \kappa^{2}
$$

where $\kappa$ is defined earlier.

Figure 4 shows the calculated $F_{\text {step }}$ and $F_{\text {plane }}$ for different values of the junction width $L$. As the energy of the incident carriers increases, both Fano factors reach a maximum around $\tilde{\epsilon}=0.03 \mathrm{eV}$, where $\tilde{\epsilon}=\epsilon_{0}+e V_{s}$ and $\tilde{\epsilon}=e V_{p}$, respectively. For higher energies, the Fano factor of the step junction oscillates around its saturation value $\frac{1}{5}$, which is five times smaller than the Fano factor expected for a Poisson process $F_{\text {Poisson }}=1$. On the other hand, the Fano factor $F_{\text {plane for }}$ the planar junction vanishes as the energy of the incident carriers increases. This can be explained by noticing that the transmission $T_{\text {plane }} \rightarrow 1$ as $\tilde{\epsilon}$ increases [see Fig. 2(b)]. This remarkable difference in the Fano factor for the two junctions again suggests that there exists an additional scattering mechanism for the step junction that does not exist in the planar junction, namely, the change of plane of propagation of the topological surface states.

Finally, we briefly comment on the impact of disorder on our results. Our calculations are robust against weak disorder that respects time-reversal (TR) symmetry. ${ }^{23}$ In this case, quantum interference patterns induced by different backscattering paths are destructive, resulting in the absence of weak localization. ${ }^{12}$ On the other hand, in the presence of TR symmetry-breaking disorder, the system exhibits localization for arbitrarily weak disorder strength, which can be characterized by the localization length $\xi_{\text {loc }}$. In this case, our calculations are valid in the limit $\xi_{\text {loc }} \gg L .^{24}$

In conclusion, we have proposed and analyzed quantum transport through a nanostep junction on the surface of a $3 \mathrm{D}$ topological insulator in the ballistic limit. Our results show that the conductance in a nanostep junction is suppressed by up to a factor of $\frac{1}{3}$ compared to similar junctions based on a single surface of a 3D topological insulator or graphene. Although the suppression depends on the ratio of the Fermi velocities and the difference in Dirac point energies of the different side surfaces of the 3D topological insulator, the saturation values of the conductance and Fano factor themselves, $G_{\text {step }} \rightarrow \frac{2}{3}$ and $F_{\text {step }} \rightarrow \frac{1}{5}$, are independent of the system parameters. We also predict oscillating behavior of the Fano factor around its saturation value. Experimental demonstration ${ }^{25}$ of our predictions will provide further insight into the scattering mechanisms involved in topological insulator nanostep junctions.

We would like to thank C. Bruder for valuable discussions. This research was supported by the Dutch Science Foundation NWO/FOM. R.P.T. acknowledges financial support by the Swiss SNF, the NCCR Nanoscience, and the NCCR Quantum Science and Technology.
${ }^{1}$ M. König et al., Science 318, 766 (2007).

${ }^{2}$ D. Hsieh et al., Nature (London) 452, 970 (2008).

${ }^{3}$ Y. Xia et al., Nat. Phys. 5, 398 (2009).

${ }^{4}$ Y. Xia et al., arXiv:0907.3089.

${ }^{5}$ M. Z. Hasan and C. L. Kane, Rev. Mod. Phys. 82, 3045 (2010).

${ }^{6}$ J. Seo et al., Nature (London) 466, 343 (2010).

${ }^{7}$ Z. Alpichshev, J. G. Analytis, J. H. Chu, I. R. Fisher, Y. L. Chen, Z. X. Shen, A. Fang, and A. Kapitulnik, Phys. Rev. Lett. 104, 016401 (2010).

${ }^{8}$ R. R. Biswas and A. V. Balatsky, Phys. Rev. B 83, 075439 (2011); D. Zhang and C. S. Ting, ibid. 85, 115434 (2012).

${ }^{9}$ Y. V. Nazarov and Y. M. Blanter, in Quantum Transport: Introduction to Nanoscience (Cambridge University Press, Cambridge, UK, 2009).

${ }^{10} \mathrm{H}$. Zhang et al., Nat. Phys. 5, 438 (2009). The parameters $A_{1}, A_{2}$, $B_{1}, B_{2}, C, D_{1}, D_{2}$, and $M$ are determined by fitting the energy spectrum of the effective Hamiltonian (1) with that of ab initio calculations.

${ }^{11}$ W.-Y. Shan, H.-Z. Lu, and S.-Q. Shen, New J. Phys. 12, 043048 (2010).

${ }^{12}$ X.-L. Qi and S.-C. Zhang, Rev. Mod. Phys. 83, 1057 (2011).

${ }^{13}$ Hexagonal warping effects of the dispersion, as studied by, e.g., L. Fu, Phys. Rev. Lett. 103, 266801 (2009), are neglected in this paper.

${ }^{14}$ C.-Y. Moon, J. Han, H. Lee, and H. J. Choi, Phys. Rev. B 84, 195425 (2011).
${ }^{15}$ E. McCann and V. I. Falko, J. Phys.: Condens. Matter 16, 2371 (2004); A. R. Akhmerov and C. W. J. Beenakker, Phys. Rev. B 77, 085423 (2008).

${ }^{16}$ D. Sen and O. Deb, Phys. Rev. B 85, 245402 (2012).

${ }^{17}$ Notice that in our case the current normal to the junction has $J_{x}$ and $J_{z}$ components and boundary conditions are given by $\left.J_{x}\right|_{x=0^{-}} ^{z=0}=$ $\left.J_{z}\right|_{x=0} ^{z=0^{+}}$and $\left.J_{z}\right|_{x=0} ^{z=L^{-}}=\left.J_{x}\right|_{x=0^{+}} ^{z=L}$.

${ }^{18}$ A. Raoux, M. Polini, R. Asgari, A. R. Hamilton, R. Fazio, and A. H. MacDonald, Phys. Rev. B 81, 073407 (2010); A. Concha and Z. Tesanovic, ibid. 82, 033413 (2010); R. Takahashi and S. Murakami, Phys. Rev. Lett. 107, 166805 (2011).

${ }^{19}$ The existence of these edge states, which decay for $x \rightarrow-\infty$ and $z \rightarrow \infty$, has been predicted in Ref. 16 .

${ }^{20}$ Note that the lower bound Eq. (11) becomes exact in the limit $\kappa \rightarrow 0$.

${ }^{21}$ G. Zhang et al., Appl. Phys. Lett. 95, 053114 (2009).

${ }^{22}$ Note that the approximation $n \gg 1$ gives a lower bound on the conductance and an upper bound on the Fano factor.

${ }^{23}$ For a recent study of the effect of potential disorder on backscattering of electrons in helical TI surface states, see X.-F. Wang, Y. Hu, and H. Guo, Phys. Rev. B 85, 241402(R) (2012).

${ }^{24}$ A. Ström, H. Johannesson, and G. I. Japaridze, Phys. Rev. Lett. 104, 256804 (2010); M. M. Glazov, E. Ya. Sherman, and V. K. Dugaev, Phys. E (Amsterdam) 42, 2157 (2010).

${ }^{25}$ As has been done for shot noise in graphene, see, e.g., L. DiCarlo, J. R. Williams, Yiming Zhang, D. T. McClure, and C. M. Marcus, Phys. Rev. Lett. 100, 156801 (2008). 